\title{
Gender and age based evaluation of clinical signs and symptoms of dengue: A secondary data analysis
}

\author{
Abubakr Momin ${ }^{1 *}$, Imtiaz Manzoor ${ }^{2}$, Natasha Mustafa $^{3}$, Zarghoona Wajid ${ }^{4}$, Ahsan Ali Siddiqui ${ }^{5}$ \\ Fareha Haleem ${ }^{6}$, Kanwal Niazi ${ }^{7}$ and Adnan Anwar ${ }^{8^{*}}$ \\ ${ }^{1}$ MBBS, Post Graduate trainee, Internal medicine, Medicine Department, Ziauddin hospital. \\ ${ }^{2}$ MBBS, FCPS, Consultant Physician, Chiniot General Hospital, Karachi. \\ ${ }^{3}$ MBBS, Lecturer, Department of Forensic Medicine, Altibri Medical College, Karachi. . \\ ${ }^{4}$ MBBS, M Assistant, Musavvir Stem cell Clinic and Pathology Laboratory, Pakistan \\ ${ }^{5}$ MBBS, Msc Public Health, Specialist Family Medicine, Ministry of Health, Riyadh. \\ ${ }^{6}$ MBBS, Women Medical Officer, Department of ENT, Civil Hospital, Karachi. \\ ${ }^{7}$ BS Research Fellow, Clinical Pathology, Dow University of Health Sciences. \\ ${ }^{8}$ MBBS, M.Phil, Assistant Professor, Department of Physiology, Al Tibri Medical College, Karachi.
}

Received: 12 January, 2019; Accepted: 14 February, 2019 ; Published: 15 February, 2019

*Corresponding author: Abubakr Momin, MBBS, Post Graduate trainee, Internal medicine, Medicine Department, Ziauddin hospital. E-mail: abubakr_ss@yahoo.com

\begin{abstract}
Background: The global incidence of dengue, a mosquito-borne viral infection, has grown dramatically in recent decades with about half of the world's population is now at risk. Its clinical manifestations include headache, fever, skin rashes, leukopenia and arthralgia. Local data evaluating age
\end{abstract} and gender based differences in clinical signs and symptoms of dengue are scarce at best.

Objective: To compare the clinical signs and symptoms of dengue across gender and age based groups.

Methods: A secondary data analysis of a randomized controlled trial on the effects of silymarin on hepatic enzymes and clinical manifestation of dengue was conducted. The clinical signs and symptoms of the dengue patients were recorded at the baseline before giving trial intervention. Chisquare test was used to make desired comparisons across gender and age based groups whereas the significance level was set at 0.05 .

Results: The study results showed that rash $(\mathrm{p}=0.047)$ and infected conjunctiva $(\mathrm{p}=0.013)$ were significantly associated with gender of the patients whereas nausea and vomiting $(\mathrm{p}=0.01)$, headache $(\mathrm{p}=0.016)$, retro-orbital pain $(\mathrm{p}=0.049)$ and infected conjunctiva $(\mathrm{p}=0.032)$ were significantly associated with age of the patients.

Conclusion: A significant association of rash and injected conjunctiva with gender of dengue patients and of nausea and vomiting, headache, retro-orbital pain and injected conjunctiva with age of dengue patients was found. Further evaluation of study findings because of their potential implications for the symptomatic management of dengue patients is recommended.

Key words: Gender; Age; Signs and symptoms; Dengue; Secondary data analysis.

\section{Introduction}

Dengue, a mosquito-borne viral infection, is found in tropical and sub-tropical climates worldwide, mostly in urban and semiurban areas. Its global incidence has grown dramatically in recent decades with about half of the world's population is now at risk [1]. In Pakistan, its epidemics have become increasingly larger over the last decade.

It has four viral strains i.e. DEN-1, DEN-2, DEN-3, and DEN-4 [2]. Majority of the cases of dengue have been estimated to be caused by DEN-2 and DEN-3 viral strains $[3,4]$.The symptoms of dengue begins with flu like illness and may lead to severe manifestations such as bleeding, sudden loss of consciousness and even loss of life [5]. Its clinical manifestations include headache, fever, skin rashes, leukopenia and arthralgia with dermatological manifestations occurring in almost $50 \%$ of cases $[6,7]$.

Diagnosis is confirmed upon detection of IgM and IgG antibodies in the patient's blood whereas ELISA is used to differentiate between primary and secondary dengue infections [8]. For rapid diagnosis of the dengue virus, NS1 antigens are detected in the patient's blood [9]. The treatment of the infection is decided on the basis of severity of clinical features and level of fluids in the body. 
Dengue is prevented by mosquitoes control and by protecting the subjects from mosquito bite [10]. Viral prevention strategies include properly disposing of waste, up gradation of water storage system and proper usage of repellents [8].

To the best of author's knowledge, local data evaluating age and gender based differences in clinical signs and symptoms of dengue are limited at best. The current study was therefore designed to compare the clinical signs and symptoms of dengue across gender and age based groups by performing a secondary data analysis.

\section{Methods}

A secondary data analysis of a randomized controlled trial, conducted at Ziauddin Hospital, Clifton, Karachi, on the effects of silymarin on hepatic enzymes and clinical manifestation of dengue was conducted after taking ethical approval. The clinical signs and symptoms of the dengue patients ( $\mathrm{n}=92)$, aged 18 to 70 years, diagnosed clinically with a positive serological test, were recorded at the baseline before giving trial intervention. The relevant data were analyzed on SPSS version 20 . Chi-square test was used to make desired comparisons across gender and age based groups whereas the significance level was set at 0.05.

\section{Results}

The mean age of the study participants was $37.36 \pm 15.54$ years, $65.2 \%$ of them were males whereas $73.9 \%$ of them were up to 45 years old.

The study results showed that rash $(\mathrm{p}=0.047)$ and infected conjunctiva $(p=0.013)$ were significantly associated with gender where male patients were more likely to have rash and infected conjunctiva than female patients $(88.3 \%$ vs. $71.9 \%$ and $61.7 \%$ vs. $34.4 \%$ respectively) (Table 1 ).

Table 1: Signs and Symptoms of Dengue: Gender based Comparison.

\begin{tabular}{|c|c|c|c|}
\hline \multirow{3}{*}{ Variables } & \multicolumn{2}{|c|}{ Gender } & \multirow{3}{*}{$\mathbf{P}$} \\
\hline & Male $(n=60)$ & Female $(n=32)$ & \\
\hline & Frequency (\%) & Frequency (\%) & \\
\hline \multicolumn{4}{|c|}{ Nausea and Vomiting } \\
\hline Yes & $43(71.7)$ & $22(68.8)$ & \multirow[t]{2}{*}{0.77} \\
\hline No & $17(28.3)$ & $10(31.2)$ & \\
\hline \multicolumn{4}{|l|}{ Body ache } \\
\hline Yes & $59(98.3)$ & $31(96.9)$ & \multirow[t]{2}{*}{$>0.999^{*}$} \\
\hline No & $1(1.7)$ & $1(3.1)$ & \\
\hline \multicolumn{4}{|l|}{ Headache } \\
\hline Yes & $50(83.3)$ & $24(75.0)$ & \multirow[t]{2}{*}{0.337} \\
\hline No & $10(16.7)$ & $8(25.0)$ & \\
\hline \multicolumn{4}{|c|}{ Retro-orbital Pain } \\
\hline Yes & $38(63.3)$ & $16(50.0)$ & \multirow[t]{2}{*}{0.216} \\
\hline No & $22(36.7)$ & $16(50.0)$ & \\
\hline \multicolumn{4}{|l|}{ Rash } \\
\hline Yes & $53(88.3)$ & $23(71.9)$ & \multirow[t]{2}{*}{0.047} \\
\hline No & $7(11.7)$ & $9(28.1)$ & \\
\hline \multicolumn{4}{|l|}{ Petechiae } \\
\hline Yes & $2(3.3)$ & $1(3.1)$ & \multirow[t]{2}{*}{$>0.999^{*}$} \\
\hline No & $58(96.7)$ & $31(96.9)$ & \\
\hline \multicolumn{4}{|c|}{ Facial Flushing } \\
\hline Yes & $46(76.7)$ & $21(65.6)$ & \multirow[t]{2}{*}{0.257} \\
\hline No & $14(23.3)$ & $11(34.4)$ & \\
\hline \multicolumn{4}{|c|}{ Infected Conjunctiva } \\
\hline Yes & $37(61.7)$ & $11(34.4)$ & \multirow[t]{2}{*}{0.013} \\
\hline No & $23(38.3)$ & $21(65.6)$ & \\
\hline
\end{tabular}


The study results further showed that nausea and vomiting $(p=0.01)$, headache $(p=0.016)$, retro-orbital pain $(p=0.049)$ and infected conjunctiva $(\mathrm{p}=0.032)$ were significantly associated with age where patients who were up to 45 years of age were more likely to have nausea and vomiting, headache, retro-orbital pain and infected conjunctiva than patients who aged 46 years or above $(77.9 \%$ vs. $50.0 \%, 86.8 \%$ vs. $62.5 \%, 64.7 \%$ vs. $41.7 \%$ and $58.8 \%$ vs. $33.3 \%$ respectively) (Table 2 ).

Table 2: Signs and Symptoms of Dengue: Age based Comparison.

\begin{tabular}{|c|c|c|c|}
\hline \multirow{3}{*}{ Variables } & \multicolumn{2}{|c|}{ Age } & \multirow{3}{*}{$\mathbf{p}$} \\
\hline & Up to 45 Years $(n=68)$ & 46 Years or Above $(n=24)$ & \\
\hline & Frequency (\%) & Frequency (\%) & \\
\hline \multicolumn{4}{|c|}{ Nausea and Vomiting } \\
\hline Yes & $53(77.9)$ & $12(50.0)$ & \multirow[t]{2}{*}{0.01} \\
\hline No & $15(22.1)$ & $12(50.0)$ & \\
\hline \multicolumn{4}{|l|}{ Body ache } \\
\hline Yes & $67(98.5)$ & $23(95.8)$ & \multirow[t]{2}{*}{$0.456^{*}$} \\
\hline No & $1(1.5)$ & $1(4.2)$ & \\
\hline \multicolumn{4}{|l|}{ Headache } \\
\hline Yes & $59(86.8)$ & $15(62.5)$ & \multirow[t]{2}{*}{0.016} \\
\hline No & $9(13.2)$ & $9(37.5)$ & \\
\hline \multicolumn{4}{|c|}{ Retro-orbital Pain } \\
\hline Yes & $44(64.7)$ & $10(41.7)$ & \multirow[t]{2}{*}{0.049} \\
\hline No & $24(35.3)$ & $14(58.3)$ & \\
\hline \multicolumn{4}{|l|}{ Rash } \\
\hline Yes & $59(86.8)$ & $17(70.8)$ & \multirow[t]{2}{*}{$0.115^{*}$} \\
\hline No & $9(13.2)$ & $7(29.2)$ & \\
\hline \multicolumn{4}{|l|}{ Petechiae } \\
\hline Yes & $3(4.4)$ & Nil & \multirow[t]{2}{*}{$0.565^{*}$} \\
\hline No & $65(95.6)$ & $24(100)$ & \\
\hline \multicolumn{4}{|c|}{ Facial Flushing } \\
\hline Yes & $53(77.9)$ & $14(58.3)$ & \multirow[t]{2}{*}{0.063} \\
\hline No & $15(22.1)$ & $10(41.7)$ & \\
\hline \multicolumn{4}{|c|}{ Infected Conjunctiva } \\
\hline Yes & $40(58.8)$ & $8(33.3)$ & \multirow[t]{2}{*}{0.032} \\
\hline No & $28(41.2)$ & $16(66.7)$ & \\
\hline
\end{tabular}

\section{Discussion}

The study results showed that rash and infected conjunctiva were significantly associated with gender ( $\mathrm{p}<0.05$ for both) where male patients were more likely to have them than female patients. The study results further showed that nausea and vomiting, headache, retro-orbital pain and infected conjunctiva were significantly associated with age $(\mathrm{p}<0.05$ for all)where patients who were up to 45 years of age were more likely to have them than patients who aged 46 years or above.
Literature search revealed a few local studies where gender or age based differences in prevalence of dengue have been reported. Khan E et al., in 2007 reported male gender to be significantly associated with dengue fever ( $\mathrm{OR}=14.7, \mathrm{P}=0.003$ ) [11]. Likewise, Ahmed S et al., in 2008reported that majority of children who suffered from 2006 outbreak of dengue fever in Karachi were males (54\%) [12]. Khan E et al., in 2010 also reported that $61 \%$ of patients who tested positive for dengue IgM over the 5 years of study period were males and that dengue occurs in a younger age in females than in males (24.2 years vs. 25.9 years, $p<0.001$ ) [13]. 
Locally in Pakistan, Humayoun MA et al., in 2010 reported the most common symptoms of dengue fever to be fever, myalgia, headache, nausea, skin rash, mucocutaneous hemorrhagic manifestations and ocular pain [14]. Similarly, Jahan F in 2011 reported the common clinical features of dengue to be high grade fever typically accompanied by chilliness, retro-orbicular pain, photophobia, backache, severe muscle ache, joint ache, nausea and vomiting and abdominal pain [15].

Although, as cited above, gender difference in prevalence of dengue have been reported earlier, any gender or age based differences in clinical presentation have not been reported yet. This study therefore was an attempt to evaluate whether such differences occur or not, and if they do, to what extent Interestingly, two of the clinical features recorded showed significant association with male gender whereas four of them were found to be significantly associated with younger age. Unfortunately as a thorough search did not reveal any relevant published data, a meaningful comparison with the pertinent literature could not be made as intended. Nevertheless, the presence of such differences warrants further exploration as they could have potential implications for the symptomatic management of dengue patients.

\section{Conclusion and Recommendation}

A significant association of rash and injected conjunctiva with gender of dengue patients and of nausea and vomiting, headache, retro-orbital pain and injected conjunctiva with age of dengue patients was found. The study findings warrant further evaluation because of the potential implications they may have for the symptomatic management of dengue patients.

\section{Conflict of Interests}

The authors report no conflict of interests

\section{References}

1. WHO. Dengue and Severe Dengue. November, 2018.

2. Malavige GN, Fernando S and Fernando DJ, Seneviratne SL. Dengue viral infections. Postgrad Med J. 2004; 80(948): 588-601. Doi: 10.1136/ pgmj.2004.019638.

3. Thein S, Aung MM, Shwe TN, Aye M, Zaw A and Aye K et al. Risk factors in dengue shock syndrome. Am J Trop Med Hyg. 1997; 56(5): 566-572.
4. Tang JW, Khanani MR, Zubairi AM, Lam WY, Lai F, Hashmi K et al. A wide spectrum of dengue IgM and PCR positivity post-onset of illness found in a large dengue 3 outbreak in Pakistan. J Med Virol. 2008; 80(12): 2113-2121. Doi: 10.1002/jmv.21290.

5. Ashley EA. Dengue fever. Trends in Anaesthesia and Critical Care. 2011; 1(1): 39-41.

6. Oishi K, Saito M, Mapua CA and Natividad FF. Dengue illness: clinical features and pathogenesis. J Infect Chemother. 2007; 13(3):125-133.

7. Pal T, Dutta SK, Mandal S, Saha B and Tripathi A. Differential clinical symptoms among acute phase Indian patients revealed significant association with dengue viral load and serum IFN-gamma level. J Clin Virol. 2014; 61(3): 365-370. Doi: 10.1016/j.jcv.2014.09.003.

8. Banerjee A, Paul UK and Bandyopadhyay A. Diagnosis of dengue fever: roles of different laboratory test methods. International Journal of Advances in Medicine. 2018; 5(2): 395-399.

9. Kumar A, Pandit VR, Shetty S, Pattanshetty S, Krish SN and Roy S. A profile of dengue cases admitted to a tertiary care hospital in Karnataka, southern India. Trop Doct. 2010; 40(1): 45-46. Doi: 10.1258/td.2009.080376.

10. Kumar K, Singh PK, Tomar J and Baijal S. Dengue: epidemiology, prevention and pressing need for vaccine development. Asian Pacific Journal of Tropical Medicine. 2010; 3(12): 997-1000.

11. Khan E, Siddiqui J, Shakoor S, Mehraj V, Jamil B and Hasan R. Dengue outbreak in Karachi, Pakistan, 2006: experience at a tertiary care center. Trans R Soc Trop Med Hyg. 2007; 101(11): 1114-1119.

12. Ahmed S, Arif F, Yahya Y, Rehman A, Abbas K, Ashraf S and Akram DS. Dengue fever outbreak in Karachi 2006--a study of profile and outcome of children under 15 years of age. J Pak Med Assoc. 2008; 58(1): 4-8.

13. Khan E, Kisat M, Khan N, Nasir A, Ayub S and Hasan R. Demographic and Clinical Features of Dengue Fever in Pakistan from 2003-2007: PLoS One. 2010; 5(9): e12505. Doi: 10.1371/journal.pone.0012505.

14. Humayoun MA, Waseem T, Jawa AA, Hashmi MS and akram J. Multiple dengue serotypes and high frequency of dengue hemorrhagic fever at two tertiary care hospitals in Lahore during the 2008 dengue virus outbreak in Punjab, Pakistan. Int J Infect Dis. 2010; 14 Suppl 3:e54-9. Doi: 10.1016/j.ijid.2009.10.008.

15. Jahan F. Dengue fever (DF) in Pakistan. Asia Pac Fam Med. 2011; 10(1): 1. Doi: 10.1186/1447-056X-10-1. 